\title{
Corrigendum: Co-receptor CD8-mediated modulation of T-cell receptor functional sensitivity and epitope recognition degeneracy
}

\author{
Barbara Szomolay ${ }^{1}$, Tamsin Williams $^{2}$, Linda Wooldridge $^{3}$ and Hugo Antonius van den Berg ${ }^{1}$ * \\ 1 University of Warwick, Coventry, UK \\ ${ }^{2}$ Institute of Infection and Immunity, Cardiff University School of Medicine, Cardiff, UK \\ ${ }^{3}$ Faculty of Medical and Veterinary Sciences, University of Bristol, Bristol, UK \\ *Correspondence: hugo@maths.warwick.ac.uk
}

Edited by:

Bruno Laugel, Cardiff University School of Medicine, UK

Reviewed by:

Brian M. Baker, University of Notre Dame, USA

Yoram Louzoun, Bar-llan University, Israel

Keywords: corrigendum, $T$ cell, $T$ cell receptor, $T$ cell repertoire, $T$ cell response

\section{A corrigendum on}

Co-receptor CD8-mediated modulation of T-cell receptor functional sensitivity and epitope recognition degeneracy by Szomolay B, Williams T, Wooldridge L, van den Berg HA. Front Immunol (2013) 4:329. doi:10.3389/fimmu.2013.00329

It has been kindly pointed out to us by Dr. Omer Dushek of Oxford University that the thermodynamic constraints (arising from the principle of detailed balance) impose the following condition on the parameters:

$$
\nu=\gamma_{\text {kin }} \delta
$$

which means that the parameter $v$ is fixed once $\gamma_{\text {kin }}$ and $\delta$ have each been assigned a value. The objective of the paper was to exhibit the range of qualitative behaviors that is possible when $\mathrm{pMHCI} / \mathrm{CD} 8$ kinetics interacts with TCR/pMHCI kinetics and to show how varying levels of the co-receptor at the T-cell surface may be able to modulate the functional sensitivity of the T-cell to various ligands in a differential fashion. These qualitative phenomena remain very much the same when we choose parameter values that respect the constraint $\nu=\gamma_{\text {kin }} \delta$, as shown in the corrected figures that follow below (Figures 24). It is these qualitative patterns that are currently guiding experimental research to elucidate CD8-mediated ligand focusing in the T-cell system. The main thrust of the paper is therefore unaltered.

The kinetic scheme (Figure 1) and the equations are all unaltered, except for a typographical error in the subscript of a quantity appearing in one of the equations. Specifically, equation (23) in the published text should read:

$$
W=M_{R} \lambda_{-1} \mathbb{P}_{0}^{0}+M_{X R} \lambda_{-4} \mathbb{P}_{0}^{*} .
$$

We apologize profusely for any inconvenience our oversight may have caused.

Conflict of Interest Statement: The authors declare that the research was conducted in the absence of any commercial or financial relationships that could be construed as a potential conflict of interest.

Received: 06 August 2014; accepted: 31 August 2014; published online: 16 September 2014.

Citation: Szomolay B, Williams T, Wooldridge L and van den Berg HA (2014) Corrigendum: Co-receptor CD8-mediated modulation of T-cell receptor functional sensitivity and epitope recognition degeneracy. Front. Immunol. 5:443. doi: 10.3389/fimmu.2014.00443

This article was submitted to T Cell Biology, a section of the journal Frontiers in Immunology.

Copyright $\odot 2014$ Szomolay, Williams, Wooldridge and van den Berg. This is an open-access article distributed under the terms of the Creative Commons Attribution License (CC BY). The use, distribution or reproduction in other forums is permitted, provided the original author(s) or licensor are credited and that the original publication in this journal is cited, in accordance with accepted academic practice. No use, distribution or reproduction is permitted which does not comply with these terms. 
A

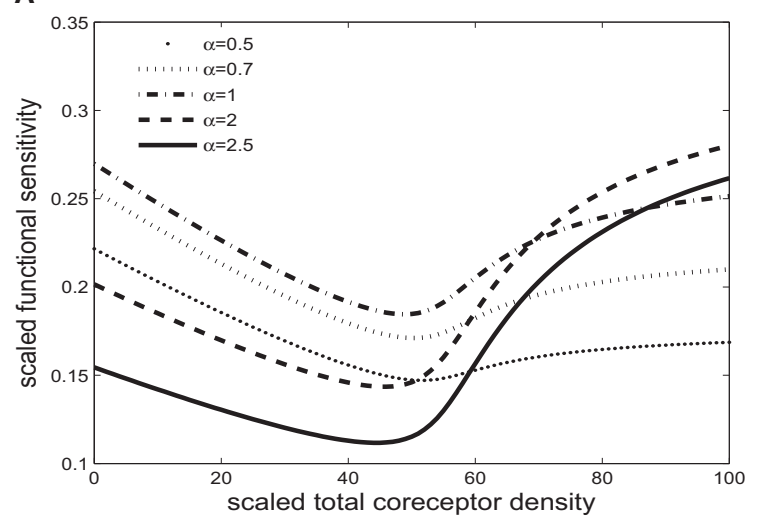

B

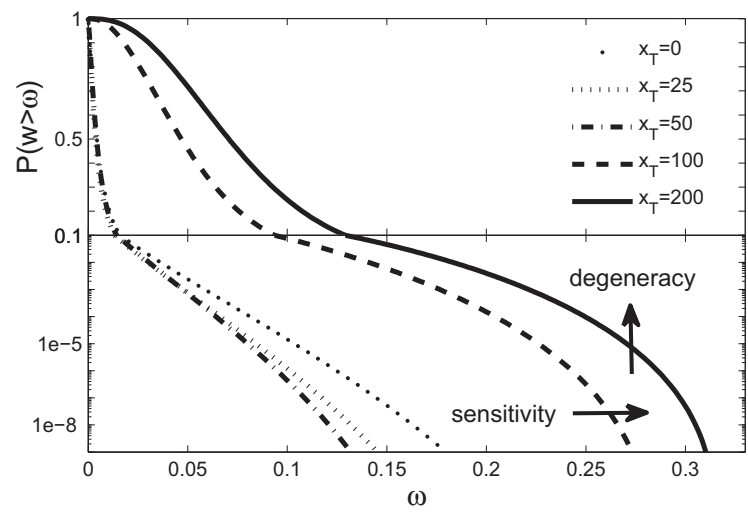

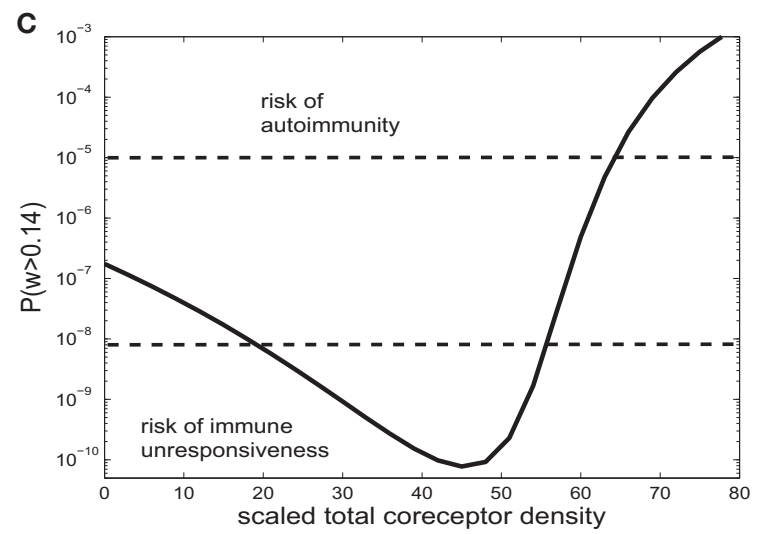

FIGURE 2 | (A) Scaled functional sensitivity $w$ as a function of scaled total CD8 density $x_{T}$ for various scaled TCR/pMHCl off-rates $\alpha$.

(B) Degeneracy curves $\mathbb{P}(w>\omega)$ for various scaled total CD8 density $x_{T}$. (C) The probability $\mathbb{P}(\mathrm{W}>\omega)$ as a function of CD8 density $x_{T}$, at a set value of functional sensitivity $\omega=0.14$. The operating range of the probability $\mathbb{P}$ is shown as a function of $x_{T}$ with dashed lines at $\mathbb{P}(w>0.14)=10^{-8}$ and $\mathbb{P}(\mathrm{W}>0.14)=10^{-5}$. Parameters are as follows: $\delta=300, n=100$, $\gamma_{\text {kin }}=0.1, \gamma_{\text {off }}=0.5, \gamma_{R}=1, \kappa=5.5, m_{T}=10, r_{T}=10$. The log-normal distribution has mean 2 and SD 0.2. Changed: $\omega=0.14, \gamma_{\mathrm{kin}}, \gamma_{\mathrm{R}}, \mathrm{K}$ and $\alpha$ 's in (A).
A

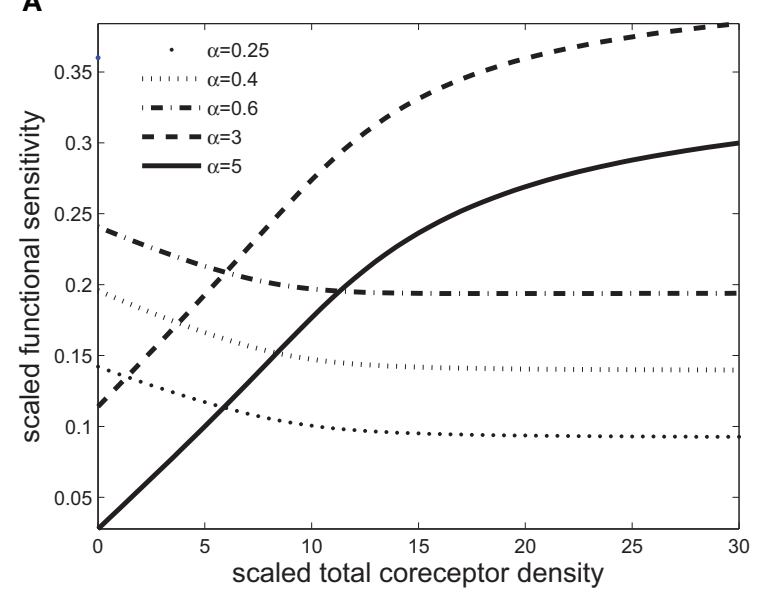

FIGURE 3 | Scaled functional sensitivity $\boldsymbol{w}$ as a function of scaled total CD8 density $\boldsymbol{x}_{\boldsymbol{T}}$. (A) Curves for various values of the scaled $\mathrm{TCR} / \mathrm{pMHCl}$ off-rate $\alpha$. (B) Curves for various values of the factor $\gamma_{R}$ by

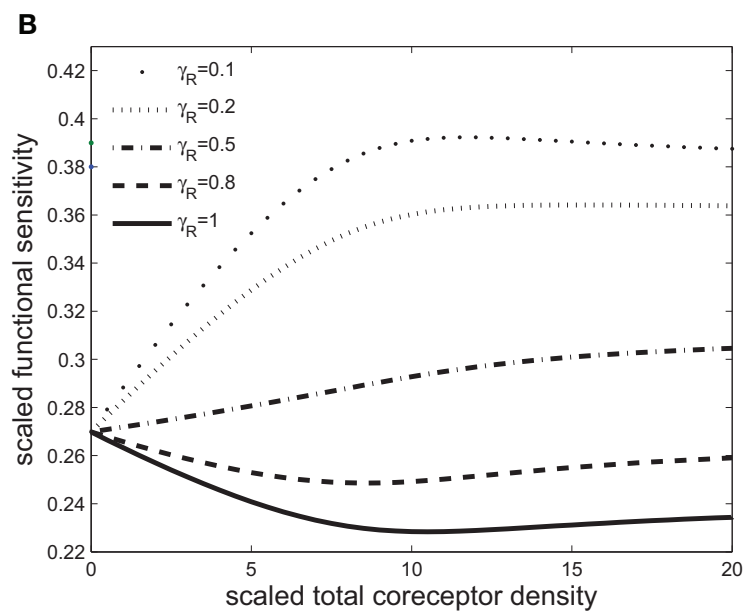

which CD8 modulates the TCR triggering threshold. Parameters, unless stated otherwise, are as follows: $\delta=2.5, n=100, \gamma_{\text {kin }}=0.5, \gamma_{\text {off }}=0.5$, $\gamma_{\mathrm{R}}=0.7, \kappa=1, m_{T}=10, r_{T}=10, \alpha=1$. Changed: $\gamma_{\mathrm{R}}$. 


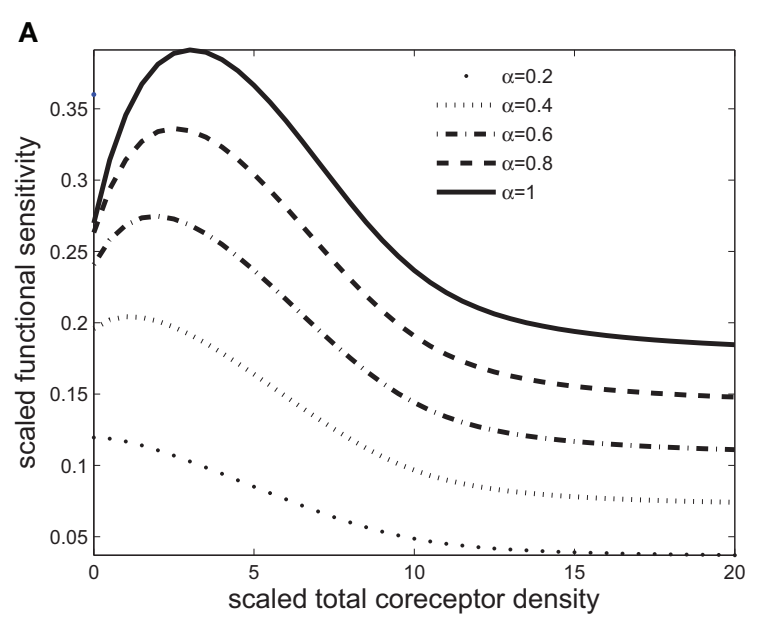

FIGURE 4 | Scaled functional sensitivity $\boldsymbol{w}$ as a function of scaled total CD8 density $\boldsymbol{x}_{\boldsymbol{T}}$. (A) Curves for various values of the scaled TCR/pMHCl off-rate $\alpha$. (B) Curves for various values of the dimensionless factor $\gamma_{\text {off }}$ expressing the modulatory effect of CD8 on

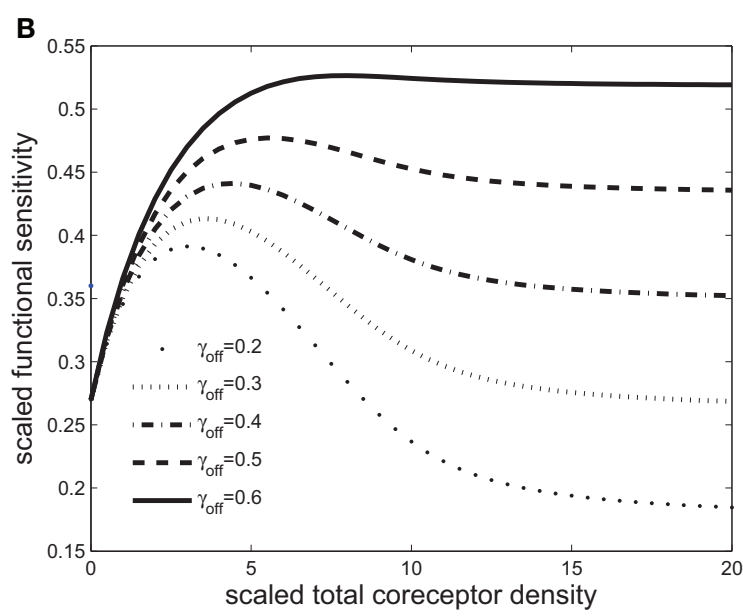

the TCR/pMHCl off-rate. Parameters, unless stated otherwise, are as follows: $\delta=500, n=100, \gamma_{\text {kin }}=0.2, \gamma_{\text {off }}=0.2, \gamma_{R}=0.01, \kappa=1$, $m_{T}=10, r_{T}=10, \alpha=1$. Changed: $\delta, \gamma_{\mathrm{kin},} \gamma_{\mathrm{R},} \alpha, \mathrm{K}$, and $\alpha$ 's in (A) and $\gamma_{\text {off }}$ 's in (B)

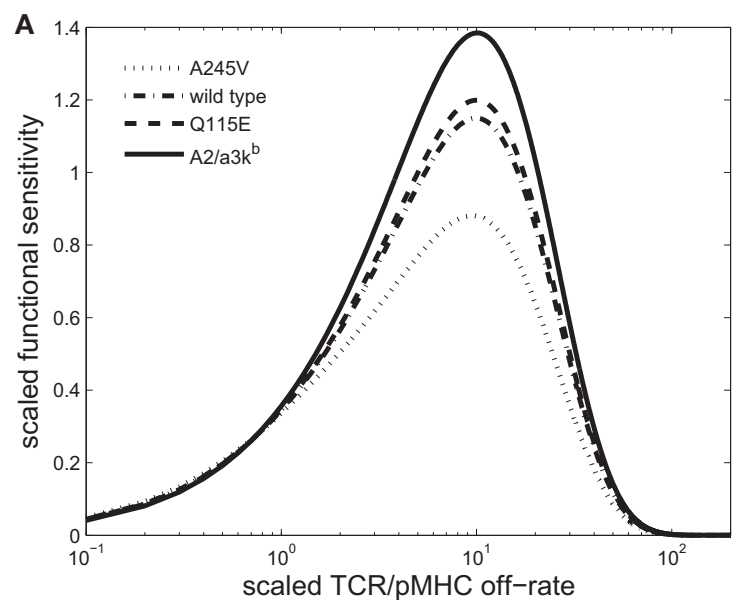

FIGURE $\mathbf{5}$ | (A) Scaled functional sensitivity $w$ as a function of scaled $\mathrm{TCR} / \mathrm{pMHCl}$ off-rate $\alpha$. (B) Degeneracy curves $\mathbb{P}(w>\omega)$ for HLA A*0201 mutants with altered binding affinity for CD8: A245V (dotted line), wild-type (semi-dashed line), Q115E (dashed line), and A2/ $\alpha 3 \mathrm{k}^{\mathrm{b}}$ (solid line). The three regions represent the overall pattern of $\mathrm{CD} 8^{+} \mathrm{T}$-cell antigen specificity and the arrow indicates the strength of $\mathrm{pMHCl} / \mathrm{CD} 8$ interaction. Wild-type parameters are as follows: $\delta=0.2, n=100$,

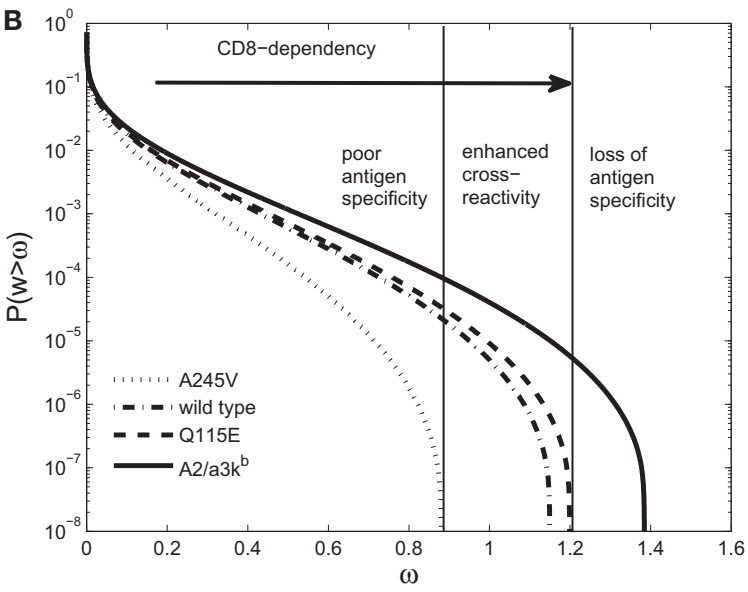

$\gamma_{\mathrm{kin}}=0.5, \gamma_{\text {off }}=0.5, \gamma_{\mathrm{R}}=0.2, \kappa=1, m_{T}=10, r_{T}=10, x_{T}=10$; the parameter $\delta$ is adjusted in proportion to the ratio between the mutant pMHCl/CD8 affinity constant (i.e., dissociation constant) and the wild-type affinity, whereas the parameters $\kappa$ and $x_{T}$ are adjusted in inverse proportion to the mutant/wild-type pMHCI/CD8 affinity (these adjustments follow from the scaling). The log-normal distribution has mean 5 and SD 0.5. Figure $5 \mathrm{C}$ has been deleted. 